\title{
Elastic Wave Scattering and Dynamic Stress Concentrations around Double Holes in Piezoelectric Media
}

\author{
Zihe Li, Haoyu Liu*, Wanzhu Zhen \\ Beihua University, Jilin, China \\ Email: ^lizihe0123@163.com
}

How to cite this paper: Li, Z.H., Liu, H.Y. and Zhen, W.Z. (2020) Elastic Wave Scattering and Dynamic Stress Concentrations around Double Holes in Piezoelectric Media. Journal of Applied Mathematics and Physics, 8, 3060-3069.

https://doi.org/10.4236/jamp.2020.812224

Received: November 26, 2020

Accepted: December 21, 2020

Published: December 24, 2020

Copyright ( 2020 by author(s) and Scientific Research Publishing Inc. This work is licensed under the Creative Commons Attribution International License (CC BY 4.0). http://creativecommons.org/licenses/by/4.0/

\begin{abstract}
In this paper, on the basis of Liu's complex function and conformal mapping methods, supplemented by local coordinate system method, e-type piezoelectric material and elastic wave scattering and dynamic stress concentrations problems with double holes question are studied, and an analytical solution is given to the problems. On the basis of multiple scattering of elastic wave theory, put forward the study about microscopic dynamics model to dynamic stress in the structure of piezoelectric composites as well as dynamic playing field. As an example, the numerical results of the dynamic stress distribution around the hole in case double equal diameter holes are given in the paper, and the influence of incident wave number and hole-spacing parameters on the dynamic stress concentration factor is analyzed.
\end{abstract}

\section{Keywords}

Dynamic Stress Concentrations, Elastic Wave Scattering, Piezoelectric, Hole

\section{Introduction}

In engineering practice, various holes of different shapes are often needed in piezoelectric materials and structures. The hole spacing will have an influence on the stress state. If the hole spacing is approximately solved according to a single hole, a large error is bound to occur.

In order to solve this problem, $\mathrm{Hu}$ [1] [2] et al. studied the diffraction and dynamic stress concentration of elastic waves with double circular holes in a plate by using complex variables and local coordinate system methods [3], and gave the function approximation sequence and boundary conditions of the general solution of the problem under the condition of incident bending waves in 
different directions. Liu [4] et al. established the integral equation of the definite solution of the problem. Finally, an example and result of the dynamic stress concentration of the interface circular hole are given, and the influence of the combination of different medium parameters on the dynamic stress concentration is discussed. Wang Ping [5] et al. discussed the relationship between the stress concentration coefficient at the defect boundary and the disturbance frequency, eccentric distance and azimuth angle, and verified the theoretical research results through numerical examples, and explained the influence of boundary and load on the concentration coefficient [6] [7]. Gao [8] et al. using boundary cannot carry out theoretical analysis and numerical calculation of elastic wave scattering and dynamic stress concentration problem of open infinite thin plate, and give the numerical results of dynamic stress concentration coefficient near the circular hole. According to the boundary conditions with zero radial and tangential stress at the edge of the hole, Chen [9] established the linear equations for solving the undetermined coefficients by the least square method, and obtained the approximate solution of the finite term coefficients. Liu [10] et al. constructed the scattering wave field by using the spherical wave potential function of expansion wave and vector shear wave. According to the boundary conditions, the boundary integral equation is established and solved by collocation, and some important laws of elastic wave scattering around three-dimensional inclusion body are revealed. And, theoretical and numerical solutions are presented for an elliptic hole or a crack in transversely isotropic piezoelectric materials under uniform internal pressure and remote electro-mechanical loads [11] [12] [13] [14] [15].

If adopting elastic dynamics theory and wave function expansion method, approximately solving the row of holes problem according to a single hole will produce larger errors. On the basis of single arbitrary hole, further solve the elastic wave scattering and dynamic stress concentrations problems in piezoelectric media with double holes.

\section{Incidence of Electroacoustic Waves and Total Electromagnetic Wave Field}

The governing equation and constitutive relation of the steady-state inverse plane dynamics problem of piezoelectrics used in this chapter are shown in (1) and (2).

$$
\begin{gathered}
4 \frac{\partial^{2} w}{\partial \zeta \partial \bar{\zeta}}+k^{2} w=0 \\
4 \frac{\partial^{2} w}{\partial \zeta \partial \bar{\zeta}}=0 \\
\tau_{x z}=c_{44}\left(\frac{\partial w}{\partial \zeta}+\frac{\partial w}{\partial \bar{\zeta}}\right)+e_{15}\left(\frac{\partial \phi}{\partial \zeta}+\frac{\partial \phi}{\partial \bar{\zeta}}\right) \\
\tau_{y z}=i c_{44}\left(\frac{\partial w}{\partial \zeta}-\frac{\partial w}{\partial \bar{\zeta}}\right)+i e_{15}\left(\frac{\partial \phi}{\partial \zeta}-\frac{\partial \phi}{\partial \bar{\zeta}}\right)
\end{gathered}
$$




$$
\begin{gathered}
D_{x}=e_{15}\left(\frac{\partial w}{\partial \zeta}+\frac{\partial w}{\partial \bar{\zeta}}\right)-\kappa_{11}\left(\frac{\partial \phi}{\partial \zeta}+\frac{\partial \phi}{\partial \bar{\zeta}}\right) \\
D_{y}=i e_{15}\left(\frac{\partial w}{\partial \zeta}-\frac{\partial w}{\partial \bar{\zeta}}\right)-i \kappa_{11}\left(\frac{\partial \phi}{\partial \zeta}-\frac{\partial \phi}{\partial \bar{\zeta}}\right) \\
\tau_{x z}+i \tau_{y z}=2 c_{44} \frac{\partial w}{\partial \bar{\zeta}}+2 e_{15} \frac{\partial \phi}{\partial \bar{\zeta}} \\
D_{x}+i D_{y}=2 e_{15} \frac{\partial w}{\partial \bar{\zeta}}-2 \kappa_{11} \frac{\partial \phi}{\partial \bar{\zeta}}
\end{gathered}
$$

Considering the case of an infinite piezoelectric material with two holes, a steady-state $\mathrm{SH}$ electroacoustic wave is incident along $\times$ axis, ignoring the time factor, the corresponding out-of-plane displacement field $w^{(i)}$ and in-plane potential field $\phi^{(i)}$ can be indicated as:

$$
\left.\begin{array}{l}
w^{(i)}=w_{0} \sum_{n=-\infty}^{\infty} i^{n} J_{n}(k|\Omega(\eta)|)\left\{\frac{\Omega(\eta)}{|\Omega(\eta)|}\right\}^{n} \\
\phi^{(i)}=\frac{e_{15}}{\kappa_{11}} w^{(i)}
\end{array}\right\}
$$

In the analysis of calculation, the local coordinate method can be used to convert the internal force component in the local polar coordinate system to the polar coordinate system to be calculated. Considering the multiple scattering between each hole, the elastic wave scattering field generated by the MTH hole in polar coordinate system $\left(r_{m}, \theta_{m}\right)$ can be described as follows:

$$
\left.\begin{array}{l}
w^{(s)}=\sum_{m=1}^{2} \sum_{n=-\infty}^{\infty} A_{n}^{m} H_{n}^{(1)}\left(k r_{m}\right) \mathrm{e}^{i n \theta_{m}} \\
\phi^{(s)}=\frac{e_{15}}{k_{11}} w^{(s)}+\sum_{m=1}^{2} \sum_{n=0}^{\infty} B_{n}^{m}\left(k r_{m}\right)^{-n} \mathrm{e}^{i n \theta_{m}}
\end{array}\right\}
$$

Of which, $A_{n}^{m}, B_{n}^{m}(m=1,2)$ are respectively scattering wave mode coefficient produced by the $m$-th hole, determined by boundary conditions.

The total wave field of the anti-plane shear wave should be superposed by the incident field and the scattering field when solving the open-hole boundary value problem of the dielectric, then the total field of the piezoelectric material with double holes is:

$$
\begin{gathered}
w^{(t)}=w^{(i)}+w^{(s)} \\
\left.\phi^{(t)}=\phi^{(i)}+\phi^{(s)}\right\} \\
w^{(t)}=\sum_{n=-\infty}^{\infty}\left[w_{0} i^{n} J_{n}(k r)+\sum_{m=1}^{2} A_{n}^{m} H_{n}^{(1)}\left(k r_{m}\right)\right] \mathrm{e}^{i n \theta_{m}} \\
\phi^{(t)}=\frac{e_{15}}{\kappa_{11}} \sum_{n=-\infty}^{\infty} \sum_{m=1}^{2}\left[w_{0} i^{n} J_{n}(k r)+\sum_{m=1}^{2} A_{n}^{m} H_{n}^{(1)}\left(k r_{m}\right)\right] \mathrm{e}^{i n \theta_{m}} \\
+\sum_{n=0}^{\infty} \sum_{m=1}^{2} B_{n}\left(k r_{m}\right)^{-n} \mathrm{e}^{i n \theta_{m}}
\end{gathered}
$$


There is no elastic displacement field in a circular hole, but only the electric potential field $\varphi^{c}$, and the charge density is zero. Therefore, the solution of this equation should satisfy the Laplace equation $\nabla^{2} \varphi^{2}=0$. Considering that the electric potential in a circular hole cannot be infinite, it should be finite, so the equation can be written as:

$$
\phi^{c}=\sum_{n=0}^{\infty} \sum_{m=1}^{2} C_{n}\left(k r_{m}\right)^{n} \mathrm{e}^{i n \theta_{m}}
$$

Then the corresponding stress can be expressed as:

$$
\begin{aligned}
T_{\rho z}= & \frac{k c_{44}}{2}(1+\lambda) \sum_{n=-\infty}^{\infty} \sum_{m=1}^{2}\left\{w _ { 0 } i ^ { n } \left[\frac{\eta_{m}}{\rho_{m}} \frac{\Omega^{\prime}\left(\eta_{m}\right)}{\mid \Omega^{\prime}\left(\eta_{m}\right)} J_{n-1}\left(k\left|\Omega\left(\eta_{m}\right)\right|\right)\left\{\frac{\Omega\left(\eta_{m}\right)}{\left|\Omega\left(\eta_{m}\right)\right|}\right\}^{n-1}\right.\right. \\
& \left.-\frac{\overline{\eta_{m}}}{\rho_{m}} \frac{\overline{\Omega^{\prime}\left(\eta_{m}\right)}}{\left|\Omega^{\prime}\left(\eta_{m}\right)\right|} J_{n+1}\left(k\left|\Omega\left(\eta_{m}\right)\right|\right)\left\{\frac{\Omega\left(\eta_{m}\right)}{\left|\Omega\left(\eta_{m}\right)\right|}\right\}^{n+1}\right] \\
& +A_{n}\left[\frac{\eta_{m}}{\rho_{m}} \frac{\Omega^{\prime}\left(\eta_{m}\right)}{\Omega^{\prime}\left(\eta_{m}\right) \mid} H_{n-1}^{(1)}\left(k\left|\Omega\left(\eta_{m}\right)\right|\right)\left\{\frac{\Omega\left(\eta_{m}\right)}{\left|\Omega\left(\eta_{m}\right)\right|}\right\}^{n-1}\right. \\
& \left.\left.-\frac{\eta_{m}}{\rho_{m}} \frac{\overline{\Omega^{\prime}\left(\eta_{m}\right)}}{\left|\Omega^{\prime}\left(\eta_{m}\right)\right|} H_{n+1}^{(1)}\left(k\left|\Omega\left(\eta_{m}\right)\right|\right)\left\{\frac{\Omega\left(\eta_{m}\right)}{\left|\Omega\left(\eta_{m}\right)\right|}\right\}^{n+1}\right]\right\} \\
& -e_{15} \sum_{n=0}^{\infty} \sum_{m=1}^{2} B_{n} n k^{-n} \frac{\overline{\eta_{m}}}{\rho_{m}} \frac{\Omega^{\prime}\left(\eta_{m}\right)}{\left|\Omega^{\prime}\left(\eta_{m}\right)\right|}\left(\overline{\Omega\left(\eta_{m}\right)}\right)^{-n-1} \\
D_{\rho}= & e_{15} \frac{\partial w}{\partial r}-\kappa_{11} \frac{\partial \phi}{\partial r}=\kappa_{11} \sum_{n=0}^{\infty} \sum_{m=1}^{2} B_{n} n k^{-n} \frac{\frac{\eta_{m}}{\rho_{m}} \frac{\Omega^{\prime}\left(\eta_{m}\right)}{\mid \Omega^{\prime}\left(\eta_{m}\right)}\left(\overline{\Omega\left(\eta_{m}\right)}\right)^{-n-1}}{D_{\rho}^{c}=-\kappa_{0} \phi^{c}=-\kappa_{0} \sum_{n=0}^{\infty} \sum_{m=1}^{2} C_{n} n k^{n} \frac{\eta_{m}}{\rho_{m}} \frac{\overline{\Omega^{\prime}\left(\eta_{m}\right)}}{\mid \Omega^{\prime}\left(\eta_{m}\right)}\left(\overline{\Omega\left(\eta_{m}\right)}\right)^{-n-1}}
\end{aligned}
$$

Of which, $\lambda=\frac{e_{15}^{2}}{c_{44} \kappa_{11}}$ is dimensionless piezoelectric constant, $\kappa_{0}$ is dielectric constant in vacuum.

\section{Boundary Conditions and Model Coefficients in the Case of Double Holes}

Piezoelectric medium with a single arbitrary hole is studied. On $\eta$ plane, the open hole, stress free, potential and normal electric displacement are set as the free boundary conditions, 6 boundary conditions can be given.

$$
\begin{aligned}
& \left.\tau_{\rho_{m} z}\right|_{\rho_{1}=a_{1}}=0 \\
& \left.D_{\rho_{m}}\right|_{\rho_{1}=a_{1}}=\left.D_{\rho_{m}}^{c}\right|_{\rho_{1}=a_{1}} \\
& \left.\phi\right|_{\rho_{m}=a_{1}}=\phi^{c} \\
& \left.\tau_{\rho_{m} z}\right|_{\rho_{1}=a_{2}}=0 \\
& \left.D_{\rho_{m}}\right|_{\rho_{2}=a_{2}}=\left.D_{\rho_{m}}^{c}\right|_{\rho_{2}=a_{2}} \\
& \left.\phi\right|_{\rho_{m}=a_{2}}=\phi^{c}
\end{aligned}
$$


Of which, $a_{1}, a_{2}$ are the radii of the double holes.

Substitute Formulas (6), (7) into the opening boundary condition Formula (11), According to the orthogonality of the function system, the six mode coefficients $A_{n}^{1}, B_{n}^{1}, C_{n}^{1}, A_{n}^{2}, B_{n}^{2}, C_{n}^{2}$ to be solved can be determined by:

$$
\begin{aligned}
& \sum_{j=1}^{6} \sum_{n=-\infty}^{\infty} E_{n}^{i} X_{n}=E_{i} \quad(i=1,2,3,4,5,6) \\
& E_{n}=\left[\begin{array}{llllll}
E_{11}^{n} & E_{12}^{n} & E_{13}^{n} & E_{14}^{n} & E_{15}^{n} & E_{16}^{n} \\
E_{21}^{n} & E_{22}^{n} & E_{23}^{n} & E_{24}^{n} & E_{25}^{n} & E_{26}^{n} \\
E_{31}^{n} & E_{32}^{n} & E_{33}^{n} & E_{34}^{n} & E_{35}^{n} & E_{36}^{n} \\
E_{41}^{n} & E_{42}^{n} & E_{43}^{n} & E_{44}^{n} & E_{45}^{n} & E_{46}^{n} \\
E_{51}^{n} & E_{52}^{n} & E_{53}^{n} & E_{54}^{n} & E_{55}^{n} & E_{56}^{n} \\
E_{61}^{n} & E_{62}^{n} & E_{63}^{n} & E_{64}^{n} & E_{65}^{n} & E_{66}^{n}
\end{array}\right], X_{n}=\left[\begin{array}{l}
A_{n}^{1} \\
B_{n}^{1} \\
C_{n}^{1}, E_{i}^{2}, \quad E_{n} \\
A_{n}^{2} \\
B_{n}^{2} \\
C_{n}^{2} \\
E_{2} \\
E_{3} \\
E_{4} \\
E_{5} \\
E_{6}
\end{array}\right.
\end{aligned}
$$

Multiply $\exp \left(-i s \theta_{m}\right)$ by both ends of Formula (12), and integrate on interval $(-\pi, \pi)$, get infinite algebraic equations as follows:

$$
\sum_{n=-\infty}^{\infty} E_{n s} X_{n}=E_{s}
$$

Of which, $E_{n s}=\frac{1}{2 \pi} \int_{-\pi}^{\pi} E_{n} \exp (-i s \theta j) \mathrm{d} \theta j, E_{s}=\frac{1}{2 \pi} \int_{-\pi}^{\pi} E_{i} \exp (-i s \theta j) \mathrm{d} \theta j$.

\section{Dynamic Stress Concentration Factor}

According to the definition of dynamic stress concentration in the opening, the dynamic stress concentration coefficient is the ratio between the annular dynamic stress around the opening and the annular stress amplitude of the incident wave in the direction of incidence, i.e.

$$
\begin{aligned}
T_{\theta z}= & \frac{i k}{2}(1+\lambda) \sum_{n=0}^{\infty}\left\{\left[\varepsilon_{n} \frac{\eta_{m}}{\rho_{m}} \frac{\Omega^{\prime}\left(\eta_{m}\right)}{\Omega^{\prime}\left(\eta_{m}\right) \mid} J_{n-1}\left(k\left|\Omega\left(\eta_{m}\right)\right|\right)\left\{\frac{\Omega\left(\eta_{m}\right)}{\left|\Omega\left(\eta_{m}\right)\right|}\right\}^{n-1}\right.\right. \\
& \left.-\frac{\overline{\eta_{m}}}{\rho_{m}} \frac{\overline{\Omega^{\prime}\left(\eta_{m}\right)}}{\Omega^{\prime}\left(\eta_{m}\right) \mid} J_{n+1}\left(k\left|\Omega\left(\eta_{m}\right)\right|\right)\left\{\frac{\Omega\left(\eta_{m}\right)}{\left|\Omega\left(\eta_{m}\right)\right|}\right\}^{n+1}\right] \\
& +\sum_{m=1}^{2} A_{n}^{m}\left[\frac{\eta_{m}}{\rho_{m}} \frac{\Omega^{\prime}\left(\eta_{m}\right)}{\Omega^{\prime}\left(\eta_{m}\right) \mid} H_{n-1}^{(1)}\left(k\left|\Omega\left(\eta_{m}\right)\right|\right)\left\{\frac{\Omega\left(\eta_{m}\right)}{\left|\Omega\left(\eta_{m}\right)\right|}\right\}^{n-1}\right. \\
& +\frac{\eta_{m}}{\rho_{m}} \frac{\left.\left.\frac{\Omega^{\prime}\left(\eta_{m}\right)}{\Omega^{\prime}\left(\eta_{m}\right) \mid} H_{n+1}^{(1)}\left(k\left|\Omega\left(\eta_{m}\right)\right|\right)\left\{\frac{\Omega\left(\eta_{m}\right)}{\left|\Omega\left(\eta_{m}\right)\right|}\right\}^{n+1}\right]\right\}}{+} \\
& +i e_{15} \sum_{n=0}^{\infty} \sum_{m=1}^{2} B_{n} n k^{-n} \frac{\eta_{m}}{\rho_{m}} \frac{\Omega^{\prime}\left(\eta_{m}\right)}{\left|\Omega^{\prime}\left(\eta_{m}\right)\right|}\left(\overline{\Omega\left(\eta_{m}\right)}\right)^{-n-1}
\end{aligned}
$$

Among it, the comparison between maximum stress factor and static in the formula is the largest dynamic stress concentration factor.

\section{Numerical Example}

With steady wave $w^{(i)}$ incidence along $\mathrm{x}$ axis, For Circular aperture mapping 
function with radius $a_{1}=a_{2}=a$, it can be taken as:

$$
\Omega=a \eta
$$

According to the formula of elastic wave scattering and dynamic stress concentration in piezoelectric materials with two holes, taking circular holes as an example, the corresponding calculation program is worked out, and $n=15$, Poisson $/ v=0.3$, and dimensionless wave number $K a=2.0-5.0$.

Figure 1 and Figure 2 respectively describe the distribution of dynamic stress concentration coefficient along a single circular hole with different parameters when the incident wave number $K a=2.0$ and $K a=5.0$. Figures 3-8 respectively describe the hole edge distribution of dynamic stress concentration coefficient along double circular hole $\mathrm{A},=\mathrm{A} 2=\mathrm{A}$ with different parameters when the spacing between the two holes is DLA $=2.1,3.0$ and 12.0 (i.e. the nearest distance between the two holes is $0.1 \mathrm{a}, 1.0 \mathrm{a}$ and $10.0 \mathrm{a}$ respectively) under the condition of incident wave number $K a=2.0$ and $K a=5.0$.

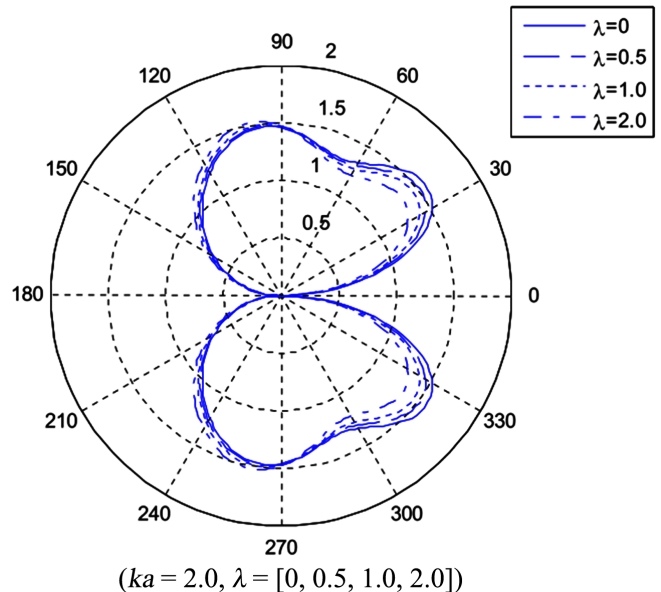

Figure 1. Dynamic stress concentration factor of single hole under different piezoelectric constants 1 .

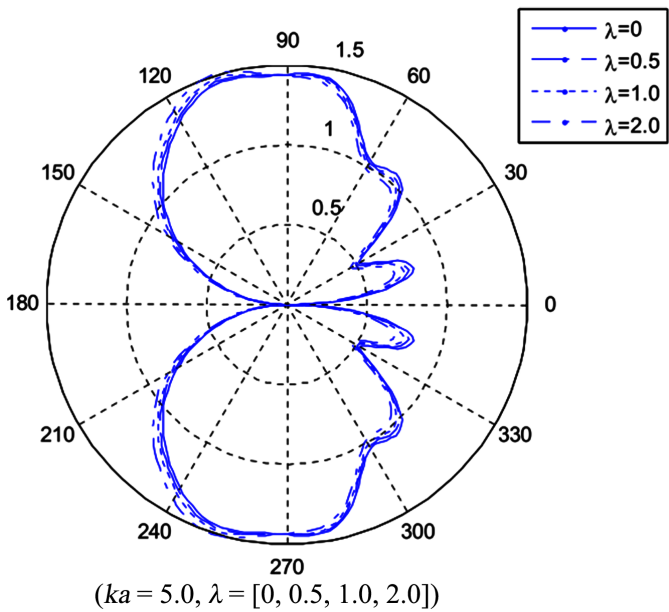

Figure 2. Dynamic stress concentration factor of single hole under different piezoelectric constants 2 . 


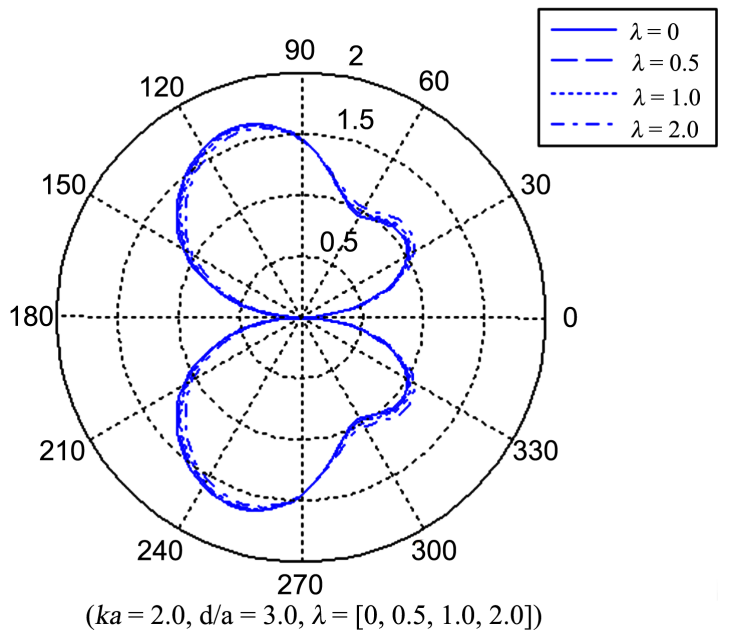

Figure 3. Dynamic stress concentration factor of double holes under different piezoelectric constants 3 .

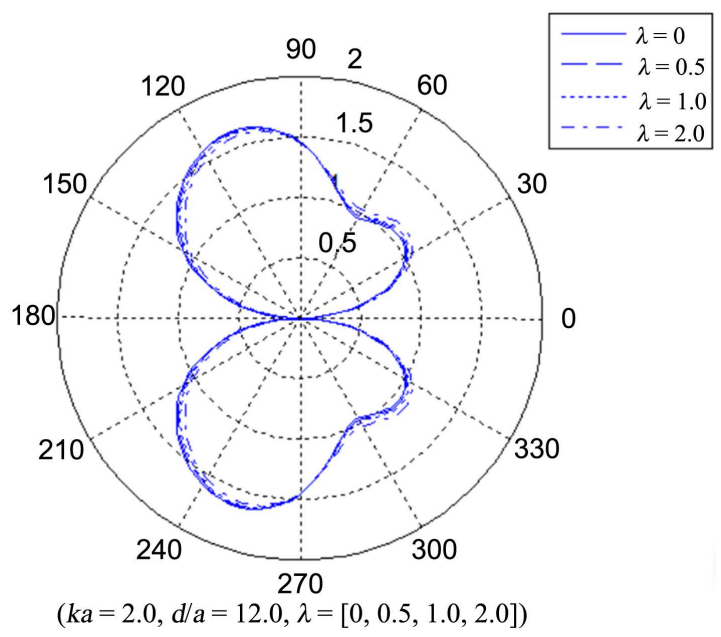

Figure 4. Dynamic stress concentration factor of double holes under different piezoelectric constants 4 .

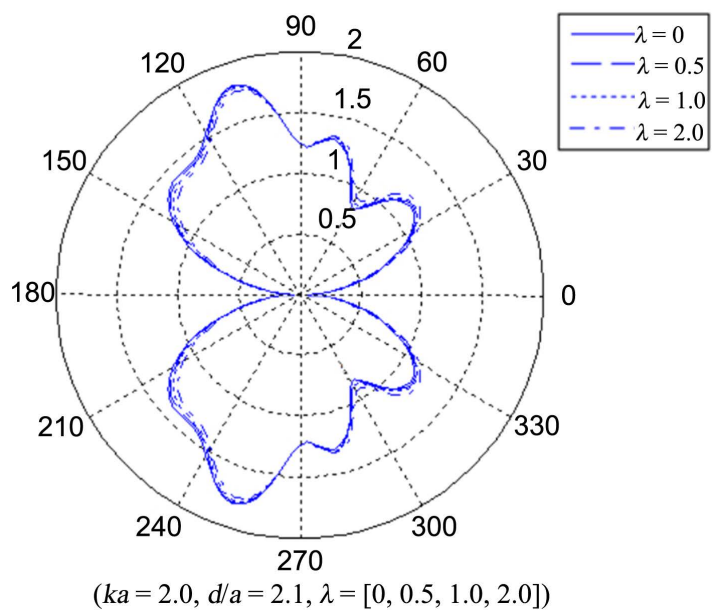

Figure 5. Dynamic stress concentration factor of double holes under different piezoelectric constants 5 . 


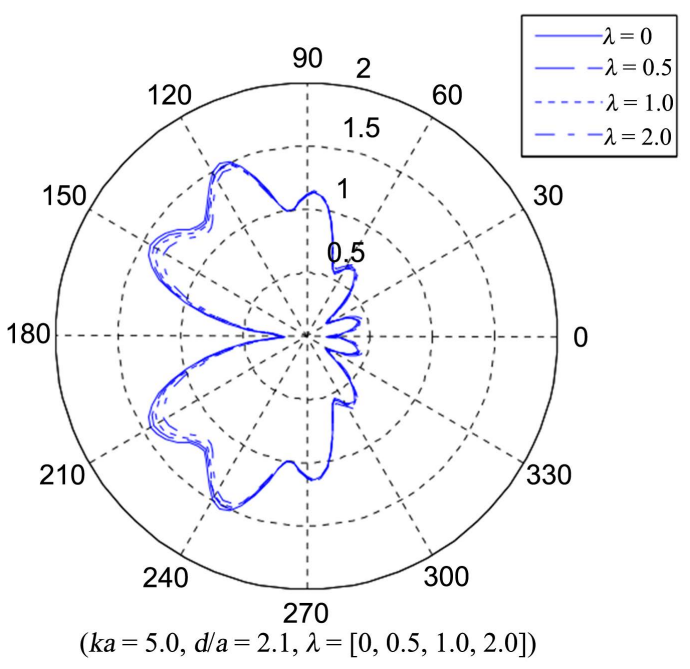

Figure 6. Dynamic stress concentration factor of double holes under different piezoelectric constants 6 .

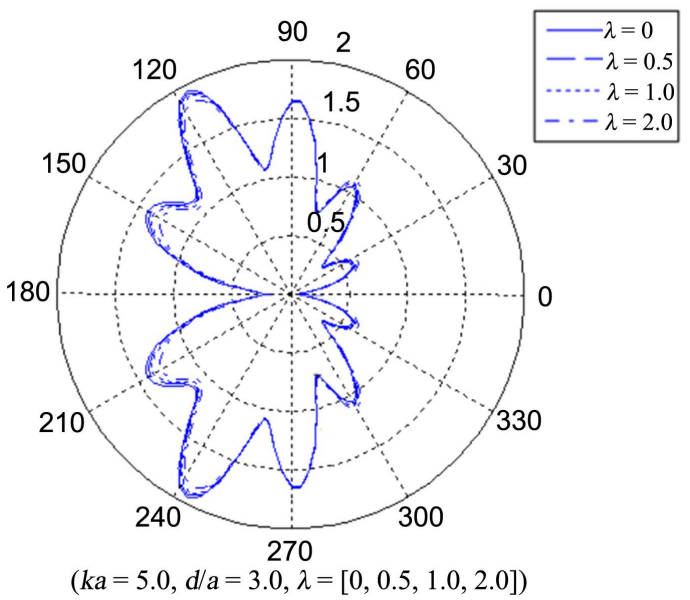

Figure 7. Dynamic stress concentration factor of double holes under different piezoelectric constants 7 .

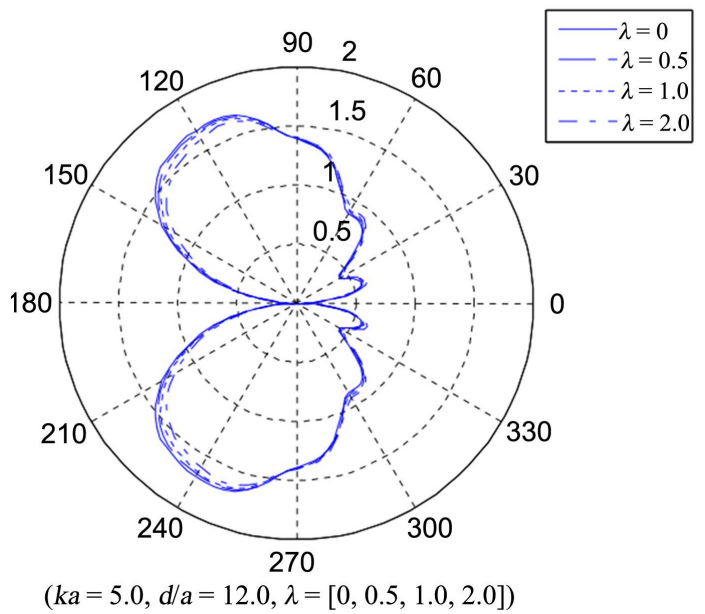

Figure 8. Dynamic stress concentration factor of double holes under different piezoelectric constants 8 . 


\section{Conclusion}

This paper, based on elastic dynamics theory, uses Liu's complex function and conformal mapping methods, and studies e-type piezoelectric material and elastic wave scattering and dynamic stress concentrations problems with double holes question. An analytical solution to the problems and numerical calculation results is given. By analysis and calculation results: 1) when the wave number $k a$ is a constant, the maximum of the dynamic stress concentration factor varies as the hole spacing $d / a$ varies, with different parameter $\lambda$. Small hole spacing corresponds to greater impact on the dynamic stress concentration factor, and large hole spacing corresponds to less impact on the dynamic stress concentration factor. When the hole spacing $d / a=12.0$, the dynamic stress concentration factor is no longer influenced by the adjacent holes, it is almost the same as the impact of a single object; 2) when the hole spacing $d / a$ is a constant, as the wave number $k a$ increases, the impact of the hole spacing on the dynamic stress concentration factor grows.

\section{Conflicts of Interest}

The authors declare no conflicts of interest regarding the publication of this paper.

\section{References}

[1] Hu, C., Ma, X.R. and Huang, W.H. (1998) Elastic Wave Scattering and Dynamic Stress Analysis of Plates with Double Circular Holes. Chinese Journal of Theoretical and Applied Mechanics, No. 5, 3-5. (In Chinese)

[2] Liu, D.K. and Hu, C. (1996) Scattering of Flexural Waves and Dynamic Stress Concentrations in Mindlin's Thick Plates with a Cutout. Acta Mechanica Sinica, 12, 169-185. https://doi.org/10.1007/BF02486795

[3] Wang, X.D. and Meguid, S.A. (2000) Modelling and Analysis of the Dynamic Behaviour of Piezoelectric Materials Containing Interacting Cracks. Mechanics of Materials, 32, 723-737. https://doi.org/10.1016/S0167-6636(00)00043-0

[4] Liu, D.K. and Liu, H.W. (1998) SH-Wave Scattering and Dynamic Stress Concentration near the Interface Circular Hole. Chinese Journal of Theoretical and Applied Mechanics, No. 5, 3-5.

[5] Wang, P. and Jiang, S.T. (2015) Scattering of SH Waves by Circular Holes in a Circular Region. Journal of Mechanical Strength, 37, 18-22.

[6] Fang, X.-Q., Hu, C. and Huang, W.-H. (2007) Dynamic Stress of a Circular Cavity Buried in a Semi-Infinite Functionally Graded Piezoelectric Material Subjected to Shear Waves. European Journal of Mechanics/ A Solids, 26, 1016-1028. https://doi.org/10.1016/j.euromechsol.2007.05.003

[7] Fang, X.-Q. (2008) Multiple Scattering of Electro-Elastic Waves from a Buried Cavity in a Functionally Graded Piezoelectric Material Layer. International Journal of Solids and Structures, 45, 5716-5729. https://doi.org/10.1016/j.ijsolstr.2008.06.014

[8] Gao, S.W. and Wang, B.L. (2001) Elastic Wave Scattering and Dynamic Stress Concentration of Porous Thin Plates. Engineering Mechanics, No. 2, 14-20.

[9] Chan, Z.G. (2019) The Least Square Method Is Used to Solve the Scattering of Plane 
P Wave on the Hole. Earthquake Engineering and Engineering Vibration, 39, 106-112.

[10] Liu, Z.X. and Wang, Z.K. (2019) Three-Dimensional Scattering and Dynamic Stress Solution of Elastic Wave Based on the Basic Solution of Spherical Wave Potential Function. Rock and Soil Mechanics, 40, 2730-2738.

[11] Li, L.H., Cui, X.W. and Guo, J.H. (2020) Interaction between a Screw Dislocation and an Elliptical Hole with Two Asymmetrical Cracks in a One-Dimensional Hexagonal Quasicrystal with Piezoelectric Effect. Applied Mathematics and Mechanics (English Edition), 41, 899-908. https://doi.org/10.1007/s10483-020-2615-6

[12] Fan, S.W., Guo, J.H. and Yu, J. (2017) Anti-Plane Problem of Four Edge Cracks Emanating from a Square Hole in Piezoelectric Solids. Chinese Journal of Aeronautics, 30, 461-468. https://doi.org/10.1016/j.cja.2016.08.018

[13] Yang, X.M., et al. (2005) Electric Field Gradient Effects in Anti-Plane Problems of a Circular Cylindrical Hole in Piezoelectric Materials of $6 \mathrm{~mm}$ Symmetry. Acta Mechanica Solida Sinica, 18, 28-36.

[14] Du, Y.L., Liu, S.H., Duan, S.J. and Li, Y.Q. (2013) Electro-Elastic Fields of Piezoelectric Materials with an Elliptic Hole under Uniform Internal Shearing Forces. Chinese Journal of Mechanical Engineering, 26, 454-461. https://doi.org/10.3901/CJME.2013.03.454

[15] Liu, S.H., Shen, Y.M. and Liu, J.X. (2012) Exact Solutions for Piezoelectric Materials with an Elliptic Hole or a Crack under Uniform Internal Pressure. Chinese Journal of Mechanical Engineering, 25, 845-852. https://doi.org/10.3901/CJME.2012.04.845 